ARTíCULOS DE INVESTIGACIÓN

\title{
Incorporación de dispositivos de enseñanza- aprendizaje de los géneros orales propios de la formación inicial en derecho: El caso de la solemne oral
}

\author{
Incorporação de dispositivos de ensino-aprendizagem dos gêneros \\ orais típicos da formação inicial em direito: $O$ caso do oral solene
}

Incorporation of teaching-learning devices of the oral genres typical of initial training in law: The case of the solemn oral

\author{
María Verónica Sánchez Gibbons (iD) Karen Urrejola Corales \\ y Nelson Becerra Rojas \\ Pontificia Universidad Católica de Chile
}

\begin{abstract}
RESUMEN Debido a la relevancia del desarrollo de habilidades orales situadas tanto para la trayectoria formativa como para el ejercicio profesional del derecho, resulta fundamental incorporar dispositivos de enseñanza-aprendizaje de los géneros orales más frecuentes de dicho campo disciplinar a lo largo del currículum. Enmarcado en el ámbito de la alfabetización académica y disciplinar, en primer lugar este artículo da cuenta tanto del proceso como del producto de la descripción del género solemne oral realizada a partir de herramientas de la Lingüística Sistémico Funcional (LSF) y del enfoque etnográfico. En segundo lugar, esta investigación analiza la percepción de los estudiantes de un curso de escritura y oralidad intensiva, anclado en la malla curricular de la carrera de Derecho en una universidad tradicional chilena, sobre la transferencia del aprendizaje de dicho género a otras instancias de la carrera. Entre las conclusiones destaca la relevancia del trabajo interdisciplinario para efectos de las acciones de alfabetización disciplinar y se discuten los alcances de la descripción de géneros de una comunidad académica particular para fines educativos.
\end{abstract}

PALABRAS CLAVE Alfabetización, competencias orales, competencias específicas de una disciplina, educación superior, enseñanza y aprendizaje (procesos y metodología), y estudios jurídicos. 
RESUMO Dada a relevância do desenvolvimento de habilidades orais situadas, tanto para a trajetória de formação quanto para a prática profissional do direito, é essencial incorporar, ao currículo, dispositivos de ensino-aprendizagem dos gêneros orais mais frequentes do referido campo disciplinar. Nesse contexto, embasado no campo da alfabetização acadêmica e disciplinar, o presente estudo explica, em primeiro lugar, o processo e o produto da descrição do gênero oral solene, realizado com ferramentas da Linguística Sistêmico Funcional (LSF) e do enfoque etnográfico. Como segundo resultado, esta pesquisa analisa a percepção dos alunos de um curso intensivo de escrita e oralidade, ancorado na malha curricular da carreira de Direito em uma universidade tradicional chilena, sobre a transferência de aprendizagem desse gênero para outras instâncias do curso. Entre as conclusões destaca-se a relevância do trabalho interdisciplinar para fins de ações disciplinares de alfabetização, bem como também se discute o escopo da descrição de gêneros de uma comunidade acadêmica específica para fins educacionais.

PALAVRAS-CHAVE Literacia, Competências orais, Competências disciplinares específicas, Ensino superior, Ensino e Aprendizagem (Processos e Metodologias), Estudos jurídicos.

ABSTRACT Given the relevance of the development of situated oral skills, both for the formative trajectory and for the professional exercise of the law, it is essential to incorporate teaching-learning devices of the most frequent oral genres of said disciplinary field across the curriculum. In this context, the present study, framed in the field of academic and disciplinary literacy, gives an account, first, of the process and the product of the description of the solemn oral genre, based on tools of Systemic Functional Linguistics (SFL) and of the ethnographic approach. As a second result, this research analyzes the students' perception of an intensive writing and orality course, anchored in the curriculum of the Law degree in a traditional Chilean university, on the transfer of learning of solemn oral genre to other instances of the career. The conclusions include the relevance of interdisciplinary work for the purposes of disciplinary literacy actions, as well as the scope of the description of genres of a particular academic community for educational purposes.

KEYWORDS Literacy, oral skills, subject-specific competences, higher education, Teaching and learning (Processes and methodology), law studies.

\section{Introducción}

La relevancia de las competencias orales en la carrera de Derecho parte del supuesto de que los estudiantes, una vez egresados, deben manejar tanto las formas escritas como orales para lograr los objetivos asociados al proceso legal que su quehacer profesional exige (Palomo, 2004). Desde esta mirada, la oralidad es una habilidad altamente valorada en la carrera, lo que se ve reflejado en su enseñanza en el aula a partir 
de prácticas constantes de ejercitación como, por ejemplo, la réplica de audiencias de juicio oral o solemnes (Coloma y Agüero, 2012). Sin embargo, a pesar de que las instancias evaluativas de oralidad son parte importante en la carrera, no hay una enseñanza explícita de la apropiación de estas prácticas:

Ahora bien, esta forma de enseñar destrezas de litigación suele carecer de base epistemológica (y lingüística) y solo se limita a promover que el estudiante replique los modos, rutinas, turnos y prácticas que los abogados y jueces acostumbran realizar en las diferentes audiencias (Coloma y Agüero, 2012: 67).

De esta manera, hay un vacío en la enseñanza de estos géneros orales, lo que no solo afecta a los estudiantes en su vida académica, sino también en su quehacer laboral. Por lo tanto, es fundamental una enseñanza explícita de las prácticas orales específicas de la disciplina para los estudiantes de la carrera de Derecho.

Como consecuencia de lo anterior, el Programa de Lectura y Escritura Académicas de la Pontificia Universidad Católica de Chile (PLEA), desde la perspectiva de la Alfabetización Académica (Lea y Street, 2006; Urrejola y Vidal, en prensa), realizó un proyecto Fondo Desarrollo de la Docencia (Fondedoc) enfocado en la identificación de los géneros más relevantes en el ámbito oral producidos por los estudiantes de derecho en su formación inicial, con el fin de generar dispositivos de enseñanzaaprendizaje en los cursos de alfabetización académica para esta disciplina. Los resultados del proyecto «Incorporación de dispositivos de enseñanza- aprendizaje de los géneros orales propios de la formación inicial en derecho en el curso Desarrollo de habilidades comunicativas para abogados» reflejaron que el género oral más utilizado y transversal es la solemne. A partir de lo anterior, se incorporó la enseñanza de este género y se creó material pedagógico específico en el contexto del curso mencionado, cátedra mínima ubicada en el primer año del currículum de la carrera de Derecho de la Pontificia Universidad Católica de Chile. Esta investigación se enmarca en la intervención realizada en este curso, considerando desde el trabajo investigativo realizado previo a su implementación hasta su evaluación por parte de los estudiantes.

A partir de lo anterior, el objetivo de este artículo es describir la intervención recién mencionada y evaluarla a partir de la percepción de los estudiantes. Para esto, primero se dará cuenta de los antecedentes de la intervención, centrados en el levantamiento del género solemne oral en el marco del proyecto realizado por PLEA. En segundo lugar, se describirá la intervención realizada y, finalmente, se dará cuenta de la evaluación del impacto de la implementación del curso a partir de lo indicado por los estudiantes. 


\section{Alfabetización académica: Una herramienta para la adquisición de conocimiento disciplinar}

Actualmente, existe consenso con respecto a que el desarrollo de competencias vinculadas con las habilidades comunicativas tiene un rol central en la formación académica de los estudiantes de educación superior. Por un lado, incorporar estas competencias en el currículum fomenta el aprendizaje y, por consiguiente, el éxito académico de los estudiantes (Barbeiro, 2003; Jewitt, 2006). Por otro lado, permiten el desarrollo del pensamiento crítico y la resolución de problemas, habilidades preponderantes para el contexto académico (Condon y Kelly-Riley, 2004).

Específicamente, la investigación en el ámbito de la alfabetización académica reconoce que la lectura, la escritura y la oralidad poseen funciones que permiten a los estudiantes incorporarse en una determinada comunidad académico-disciplinar (Swales, 1990), apropiarse de los contenidos disciplinares (Miras, 2000; Ibáñez y González, 2017) y construir conocimiento en el marco de su disciplina (Schmandt-Besserat y Erard, 2008). Así, la alfabetización académica se conceptualiza como un proceso eminentemente estratégico, cuyo objetivo es articular habilidades comunicativas y conocimientos disciplinares. Al respecto, Ibáñez y González (2017: 28) desarrollan el aspecto estratégico y la dimensión sociocognitiva de este tipo de alfabetización:

Concebimos la AA [alfabetización académica] como un complejo proceso de inserción de los estudiantes universitarios en su cultura académico-disciplinar, que implica el desarrollo de estrategias discursivas diversas y especializadas, las cuales son necesarias para desenvolverse exitosamente en el ámbito académico y, posteriormente, en el profesional.

De acuerdo con los autores, este proceso está mediado por las prácticas discursivas de las disciplinas. Entonces, el diseño de intervenciones curriculares en el marco de la alfabetización debe brindar importancia a la variabilidad en las disciplinas, a los modos de pensar y conceptualizar la realidad que poseen y a las tareas de lectura, escritura y oralidad que se concretan en los géneros discursivos de la disciplina en los que se representan sus propósitos comunicativos (Jarpa y Satt, 2017).

Finalmente, la alfabetización académica representa un doble esfuerzo para las instituciones de educación superior. Por un lado, intervenir el currículum con cursos de estas características tiene como objetivo mejorar las habilidades comunicativas de los estudiantes. Por otro lado, pretende instalar la idea de que estas habilidades son un medio para la adquisición de conocimiento disciplinar. Este proceso se logra a través de las funciones asociadas a dichas habilidades: la función epistémica y la función semiótica. La primera hace referencia a que el desarrollo de habilidades comunicativas permite que los estudiantes adquieran conocimientos, se vuelvan conscientes de estos y sean capaces de autorregular sus procesos de aprendizaje (Klein y 
Boscolo, 2016). La segunda establece que el manejo de estas habilidades propicia la creación y recreación del conocimiento, logrando que los estudiantes aporten nuevo conocimiento académico-disciplinar en las comunidades en que participan (Ibáñez y González, 2017).

\section{El desarrollo de la competencia oral académica en derecho}

La competencia oral involucra el dominio de distintos sistemas que funcionan de manera paralela, como el lenguaje o discurso (lo dicho propiamente tal), el paralenguaje (aspectos fonéticos como el tono o intensidad) y la kinésica (gestos, movimientos), que se complementan entre sí para entregar el significado a la audiencia (Poyatos, 2003). De esta manera, el individuo debe ser capaz de considerar y controlar todos estos «canales» para poder realizar exposiciones orales eficientes y poder convencer a su audiencia (Doumont, 2009).

El trabajo de la oralidad en el ámbito académico presupone articular tanto los aspectos propios de la modalidad oral (dominio de distintos canales) como las convenciones particulares de cada disciplina sobre este tema, según los postulados de la alfabetización académica revisados. Así, en la oralidad jurídica, es necesario considerar que el abogado debe poder manejar los recursos de la exposición oral desde una perspectiva más exigente aún, ya que involucra maximizar todos los medios para persuadir a otros en su quehacer académico-laboral, y que la eficacia de esta competencia se mide específicamente en el impacto de su oratoria en la audiencia (Palomo, 2004).

A partir de lo anterior, es necesario que la enseñanza y evaluación de la competencia oral en derecho transparente a los estudiantes las exigencias propias de la oralidad en la labor jurídica, con el fin de que estos se vuelvan conscientes del proceso de aprendizaje y de las distintas habilidades requeridas en su propio ámbito disciplinar específico (Cuenca y Andino, 2020). Esta inquietud ha sido recogida desde distintas iniciativas en el contexto latinoamericano. Es así como es posible observar diferentes trabajos que apuntan a relevar la importancia de generar acciones curriculares que entreguen instrucción explícita sobre el discurso jurídico y, en especial, con respecto a la oralidad a los estudiantes en su formación como futuros abogados.

Este es el caso de Álvarez (2008), para quien la enseñanza del discurso jurídico, tanto el oral como el escrito, debe ser parte indispensable de la formación de todo abogado. En este contexto, propone que el trabajo de dicho objetivo formativo debe ser el fruto de la relación interdisciplinaria entre el Derecho y la Lingüística, relación en la que la segunda debe ser instrumental. Para lo anterior, la autora propone la incorporación de cursos a lo largo del currículum de derecho que apunten al desarrollo de competencias de lectura, escritura y oralidad propias del contexto de este campo disciplinar. 
También, es relevante destacar el trabajo de Coloma y Agüero (2012) quienes, desde la comunidad jurídica y anclados en los principios de la alfabetización académica, relevan la necesidad de trabajar de forma explícita el desarrollo de competencias de comprensión y producción de discursos propios del campo del derecho en el contexto de la formación de estudiantes.

Por último, también es posible consignar trabajos como el de Andino y otros (2019), en el que se da cuenta de los resultados de una herramienta pedagógica orientada a que los estudiantes puedan desarrollar esta competencia a partir de enfrentar el desafío de participar en situaciones comunicativas reales, como la veeduría.

De esta manera, es posible constatar que desde la comunidad de enseñanzaaprendizaje del derecho hay una inquietud con respecto a la enseñanza explícita de esta competencia y de su relevancia tanto para la participación en la trayectoria formativa como para el ejercicio profesional. En este contexto, este trabajo busca generar un aporte tanto a la comunidad de formadores de este campo del conocimiento como al de la alfabetización académica.

\section{Metodología}

El estudio se enmarca en la tradición cualitativa y en el paradigma de la investigación-acción, cuyo propósito es detectar una necesidad en una determinada comunidad y, mediante el análisis recursivo de datos, proponer mejoras atingentes al contexto en estudio (Israel y otros, 2008). Como se señaló, esta investigación se enmarca en la comunidad disciplinar de derecho de la Pontificia Universidad Católica de Chile y se interesa por evaluar el dispositivo de enseñanza-aprendizaje que surge de la necesidad de andamiar la comunicación académica oral en estudiantes de primer año de la disciplina. De acuerdo con Reason y Bradbury (2008), el último fin de una investigación de estas características es empoderar a los miembros de una comunidad mediante un proceso investigativo situado. En este aspecto, el estudio intenta proporcionar herramientas a los estudiantes de formación inicial de derecho para que puedan desenvolverse exitosamente en los primeros años de la inserción disciplinar a través de estrategias que propicien el desarrollo de la comunicación oral académico-disciplinar.

\section{Preguntas de investigación}

Este estudio contempló tres fases: descripción del género, diseño e implementación de estrategias de enseñanza-aprendizaje de él y evaluación de la implementación. La primera buscó dar respuesta a cuáles son los géneros orales propios de la formación inicial en derecho más frecuentes y que resultan adecuados para ser incorporados como objetivos de aprendizaje en el contexto del curso «Desarrollo de habilidades 
comunicativas para abogados». Por otra parte, la segunda fase tuvo como eje orientador responder a la pregunta relativa a las estrategias de enseñanza-aprendizaje que resultan más propicias para lograr el manejo, por parte de los estudiantes, de el o los géneros identificados para su implementación en el contexto del curso «Desarrollo de habilidades comunicativas para abogados». Por último, la tercera fase respondió a la pregunta de cómo evalúan los estudiantes el proceso de enseñanza-aprendizaje del género solemne oral en el contexto del curso indicado.

\section{Objetivos de la investigación}

El objetivo general de la investigación corresponde a describir el proceso de intervención, orientado a incorporar dispositivos de enseñanza-aprendizaje de los géneros orales propios de la formación inicial en derecho. Como objetivos específicos se presentan los siguientes:

- Identificar y caracterizar los géneros orales propios de la formación en derecho más frecuentes y que resultan adecuados para ser incorporados como objetivos de aprendizaje en el contexto del curso «Desarrollo de habilidades comunicativas para abogados».

- Diseñar estrategias de enseñanza-aprendizaje que resulten propicias para lograr el manejo por parte de los estudiantes de el o los géneros identificados y descritos en el contexto del curso «Desarrollo de habilidades comunicativas para abogados».

- Evaluar la implementación, a partir de la percepción de los estudiantes, del dispositivo de enseñanza-aprendizaje de el o los géneros orales trabajados en el marco del curso «Desarrollo de habilidades comunicativas para abogados».

\section{Fases de la investigación}

Como se señaló, la intervención aquí descrita consideró tres fases. Cabe señalar que cada una de las fases consideró la implementación de consentimientos informados, los que fueron socializados con los informantes clave (docentes, directivos y estudiantes) de forma oportuna. A continuación, se detalla cada una de ellas.

\section{Descripción del género}

Para lograr los objetivos de esta fase, se llevaron a cabo varias acciones que se detallan en la tabla 1. 
Tabla 1. Acciones emprendidas para la identificación y descripción de el o los géneros

\begin{tabular}{|ll|}
\hline Acciones & Descripción de la acción \\
\hline $\begin{array}{l}\text { Aproximación a la comunidad } \\
\text { discursiva intervenida }\end{array}$ & $\begin{array}{l}\text { Reunión con las autoridades de la Facultad de Derecho con el fin de presentar el proyecto y } \\
\text { determinar qué cursos de la malla evalúan a sus estudiantes a través de géneros orales. }\end{array}$ \\
\hline $\begin{array}{l}\text { Selección de asignaturas asociadas } \\
\text { al género }\end{array}$ & $\begin{array}{l}\text { A partir de una reunión previa, se realizó la selección de dos cursos iniciales de la malla que } \\
\text { evalúan el género solemne oral. }\end{array}$ \\
\begin{tabular}{|l} 
Recopilación de corpus \\
Se contactó a los docentes de las asignaturas correspondientes y se procedió a videograbar la \\
evaluación de las solemnes orales de dos secciones de cada uno de los cursos escogidos. De \\
ca Sistémica Funcional
\end{tabular} & $\begin{array}{l}\text { Una vez transcritos los datos, se procedió al análisis de las muestras desde la perspectiva de la } \\
\text { Lingüística Sistémico Funcional con enfoque en análisis de periodicidad y de figuras (Martin, }\end{array}$ \\
\hline $\begin{array}{l}\text { Produción Martin y Rose, 2008; Martin y Rose, 2012; Dreyfus y otros, 2016). } \\
\text { mente informados }\end{array}$ & $\begin{array}{l}\text { En articulación con el análisis antes mencionado, se realizaron entrevistas semiestructuradas a } \\
\text { los dos docentes de cada curso seleccionado en las que se buscó establecer las representaciones } \\
\text { más relevantes de los docentes en cuanto al género solemne oral. }\end{array}$ \\
\begin{tabular}{|l} 
Validación de la descripción del \\
género
\end{tabular} & $\begin{array}{l}\text { A partir de la realización de entrevistas semiestructuradas a los docentes de los cursos } \\
\text { seleccionados y de un focus group con profesores y directivos de la carrera de Derecho se buscó, } \\
\text { a partir de la presentación de los resultados obtenidos hasta el momento, la validación de la } \\
\text { descripción del género. }\end{array}$ \\
\hline
\end{tabular}

\section{Diseño e implementación de estrategias de enseñanza-aprendizaje del género}

En primer lugar, el diseño del dispositivo consideró la socialización de la descripción del género (fase 1) con el equipo docente PLEA a cargo del curso. En segundo lugar, se reformuló el programa del curso a partir de la inclusión de sesiones enfocadas a la enseñanza explícita del género, lo que involucró el diseño de una nueva secuencia pedagógica y la generación de recursos didácticos específicos.

\section{Evaluación de la implementación}

Con el objetivo de evaluar la implementación realizada, se construyó una encuesta destinada a los estudiantes del curso intervenido. Dicho cuestionario, realizado en formato online mediante la plataforma SurveyMonkey, fue enviado a la cohorte 2018 $(\mathrm{n}=320)$ y fue contestado por 178 estudiantes al finalizar el primer semestre del 2018.

El cuestionario se dividió en dos ítems: uno cerrado y otro con dos preguntas de respuesta abierta. El primero correspondía a una escala de Likert en la que los participantes debían informar su nivel de acuerdo con cuatro afirmaciones: i) En el contexto del curso «Desarrollo de habilidades comunicativas para abogados» se me entregaron lineamientos claros acerca de cómo estructurar una respuesta suficiente en una solemne oral; ii) en el contexto del curso «Desarrollo de habilidades comunicativas para abogados» se me entregaron claros lineamientos acerca de cómo desempeñarme paraverbalmente de forma suficiente en una solemne oral; iii) lo trabajado en torno al género solemne oral en el contexto del curso me sirvió para enfrentarme a las solem- 
nes orales que he tenido que enfrentar hasta el momento en los otros cursos de este semestre; y iv) la exigencia de las instancias de solemne de los otros cursos fue similar a la exigencia de la solemne oral de este curso.

El segundo ítem correspondía a dos preguntas abiertas que solicitaban a los estudiantes que señalaran los aspectos favorables y mejorables del curso. Se optó por incluir dos preguntas abiertas en el cuestionario, ya que permitiría a los participantes complementar las respuestas del primer ítem. El análisis de estos datos se hizo mediante el análisis de contenido (Bardin, 1991).

\section{Resultados}

\section{Descripción de género: Solemne oral}

Como resultado del análisis realizado en la fase 1, se identificó el género solemne oral como uno de los más frecuentes en el contexto de la formación inicial en derecho. Este género se construye a partir de una interacción verbal entre el profesor - $\mathrm{y}$ a veces el ayudante del curso- que dirige la solemne y el estudiante, que tiene la misión de responder a las preguntas que se le proponen. Esta modalidad oral e interactiva de la solemne supone, además, diferentes formas de realizarse: en algunos casos los estudiantes tienen un tiempo - aunque breve- para planificar sus respuestas en la forma de un monólogo inicial y responder algunas preguntas específicas de sus evaluadores. En otros casos, la solemne se lleva a cabo como un intercambio de preguntas y respuestas entre el profesor y el estudiante, en el que este último se limita a ofrecer la información específica que el profesor le solicita.

A pesar de estas diferencias, con respecto a cómo se construye interactivamente el género solemne oral, es posible observar una serie de patrones comunes en él. En otras palabras, es posible determinar un propósito global compartido y algunas etapas que sistemáticamente aparecen en las instancias de género solemne oral analizadas. El propósito pedagógico de este género es evaluar el conocimiento del estudiante en relación con una serie de conceptos y principios, que determinan la lógica básica que los estudiantes deben desarrollar en su formación como futuros abogados. En términos más específicos, el propósito discursivo del género solemne oral es definir y explicar un fenómeno semiótico - un concepto, un principio, una idea- a partir de una serie de recursos específicos.

Para alcanzar este propósito discursivo, el género solemne oral se organiza a partir de al menos cuatro etapas claramente distinguibles: Establecimiento del fenómeno, fenómeno, clasificación del fenómeno (optativa) y aplicación del fenómeno. Estas etapas se pueden ver en la tabla 2. A continuación, se describe cada una de ellas a partir del análisis de un ejemplo del corpus.

Junto con la descripción de las etapas, fue posible observar el uso de distintos recursos discursivos que permiten la construcción del género: marcadores discursivos, 
Tabla 2. Etapas del género solemne oral

\begin{tabular}{|c|c|}
\hline Etapa 1 & Establecimiento del fenómeno \\
\hline Descripción & $\begin{array}{l}\text { Corresponde a la pregunta o solicitud de información que hace el profesor al estudiante para dar inicio a la } \\
\text { solemne. Esta etapa puede realizarse de manera oral, como parte de la interacción o en otra modalidad como, } \\
\text { por ejemplo, a partir de papeles en los que hay temas o preguntas escritas que los estudiantes seleccionan. De } \\
\text { todas maneras, esta etapa es esencial para dar inicio al género debido a que establece el tema o campo del que se } \\
\text { tratará la solemne y las expectativas del profesor con respecto a esta tarea. }\end{array}$ \\
\hline $\begin{array}{l}\text { Ejemplo de un } \\
\text { estudiante }\end{array}$ & $\begin{array}{l}\text { (El estudiante ha preparado su respuesta a partir de la selección de un tema en un papel entregado por el profesor } \\
\text { o bien a partir de una pregunta hecha por el profesor directamente) } \\
\text { E: La ley eterna. }\end{array}$ \\
\hline Etapa 2 & Fenómeno \\
\hline Descripción & $\begin{array}{l}\text { Se presenta el concepto, idea o principio alrededor del que se organiza la instancia del género solemne. En esta } \\
\text { etapa, el recurso central es la definición del fenómeno. Para construir una definición apropiada del fenómeno } \\
\text { en cuestión es esencial el uso del lenguaje especializado propio de la disciplina, ya que es posible que aparezcan } \\
\text { términos que en otros ámbitos tengan un significado de sentido común (como el concepto de «obligación»), pero } \\
\text { que en el ámbito del derecho poseen un sentido especializado y que, por lo tanto, deben ser claros y especializa- } \\
\text { damente definidos. Esto explica también la complejidad del proceso de definición que se observa en esta etapa, } \\
\text { ya que no solo se define el término inicial, sino que también se describen o explican los términos que componen } \\
\text { esa definición. Esto permite asegurar que el fenómeno definido sea construido en términos especializados, } \\
\text { apropiados al campo del derecho. }\end{array}$ \\
\hline $\begin{array}{l}\text { Ejemplo de un } \\
\text { estudiante }\end{array}$ & $\begin{array}{l}\text { La ley eterna. ¿Qué es la ley eterna? ¿Cómo la definimos? La definimos como la razón de la divina sabiduría en } \\
\text { cuanto dirige a todos los entes a su último fin. Ya en esta definición nos encontramos un problema, ya que [esta] } \\
\text { divina sabiduría se relaciona con Dios y Dios de por sí no es evidente, hay que demostrarlo, por lo cual vamos a } \\
\text { recurrir a las vías que Santo Tomás ocupa para demostrar su existencia, en específico a la quinta vía. }\end{array}$ \\
\hline Etapa 3 & Clasificación del fenómeno \\
\hline Descripción & $\begin{array}{l}\text { Una vez que el fenómeno alrededor del cual se desarrolla ha sido definido, explicado y descrito completamente, } \\
\text { y solo si la pregunta o el tema en cuestión lo exige, ocurre una tercera etapa que supone el desarrollo de taxono- } \\
\text { mías vinculadas al concepto inicial o a los términos de su definición. Dicho en otras palabras, la tercera etapa del } \\
\text { género solemne consiste en respuestas a preguntas en que se solicita una clasificación o tipos de. En esta etapa el } \\
\text { recurso fundamental es el de la clasificación. }\end{array}$ \\
\hline $\begin{array}{l}\text { Ejemplo de un } \\
\text { estudiante }\end{array}$ & $\begin{array}{l}\text { Lo podemos clasificar en circunstancias esenciales y circunstancias accidentales, y es bien importante porque las } \\
\text { circunstancias esenciales se integran, de alguna forma, a otra fuente de la moralidad que es el objeto moral. }\end{array}$ \\
\hline Etapa 4 & Aplicación del fenómenow \\
\hline Descripción & $\begin{array}{l}\text { Una vez que el fenómeno alrededor del cual se desarrolla ha sido definido, explicado y descrito completamente, } \\
\text { el estudiante debe dar cuenta de que puede aplicar y ejemplificar el fenómeno en cuestión. Generalmente, los } \\
\text { estudiantes utilizan objetos o situaciones de la vida cotidiana como ejemplo para explicar en términos simples un } \\
\text { concepto complejo o abstracto. Esta es una etapa altamente valorada por los docentes de la Facultad de Derecho, } \\
\text { ya que da cuenta de que el estudiante comprende realmente el fenómeno. }\end{array}$ \\
\hline $\begin{array}{l}\text { Ejemplo de un } \\
\text { estudiante }\end{array}$ & $\begin{array}{l}\text { La circunstancia esencial es de tal magnitud que no puede pasar desapercibida, de alguna forma, al hombre } \\
\text { prudente, por lo cual puede transformar un objeto que era bueno a uno malo, y es muy [...] Voy a dar un ejemplo } \\
\text { para que se pueda entender. [Siendo] todas las fuentes de la moralidad una intención recta, divertirme, por ejem- } \\
\text { plo, un objeto moral bueno: escuchar música. Yo [planeo] escuchar música para divertirme. Sin embargo, hay una } \\
\text { circunstancia esencial, que voy a escuchar música a las cuatro de la mañana con un equipo de música muy amplio } \\
\text { que podría despertar a mis vecinos. Esta circunstancia, a mi parecer, es esencial, ya que modifica la bondad del } \\
\text { objeto moral que a un principio era bueno. Ahora se transforma en malo. Por otro lado, las que podemos conside- } \\
\text { rar como verdaderas circunstancias [¿?] que circundan al mismo acto y tienen solamente el poder de aumentar } 0 \\
\text { disminuir la malicia del acto son las circunstancias accidentales. }\end{array}$ \\
\hline
\end{tabular}


intertextualidad y ejemplos de sentido común. Los marcadores discursivos («primero me referiré a», «para terminar», «a modo de ejemplo»), propician la organización del texto oral con el objetivo de que sea más comprensible para la audiencia. En cuanto a la intertextualidad como recurso, los estudiantes hacen uso de esta para introducir voces de autoridades en el campo (típicamente, autores de textos clave o leyes) para construir la definición y/o la clasificación del fenómeno. Finalmente, el uso de ejemplos de sentido común es un recurso que permite al estudiante transferir el contenido que se le solicita en la evaluación a situaciones cotidianas, en las que podría aplicarse el concepto, principio o idea.

\section{Diseño e implementación de estrategias de enseñanza-aprendizaje del género}

La descripción del género permitió la reestructuración del curso «Desarrollo de habilidades comunicativas para abogados». Este proceso consideró dos acciones consecutivas: reformulación del programa del curso y diseño de la secuencia didáctica para la enseñanza y la evaluación del género solemne oral.

La primera acción consideró la reformulación del programa del curso al incorporar dentro de los objetivos de aprendizaje el desarrollo de las habilidades comunicativas orales a partir del trabajo del género solemne oral. Asimismo, se agregó una unidad específica para la enseñanza y la evaluación explícita de este género.

La segunda acción consistió en el diseño de una nueva secuencia de enseñanzaaprendizaje idónea para el trabajo con este nuevo género, considerando a su vez los nuevos recursos pedagógicos creados y las propuestas de la LSF. Esta reestructuración del programa siguió la secuencia que se expone en la figura 1.

Como se puede ver en la figura 1, la secuencia pedagógica comienza con la construcción guiada del campo, acción basada en la enseñanza sobre el contexto del género como práctica académica y social. Como segundo elemento, se presenta la enseñanza explícita del género: estructura, contenido y realización. Un tercer elemento

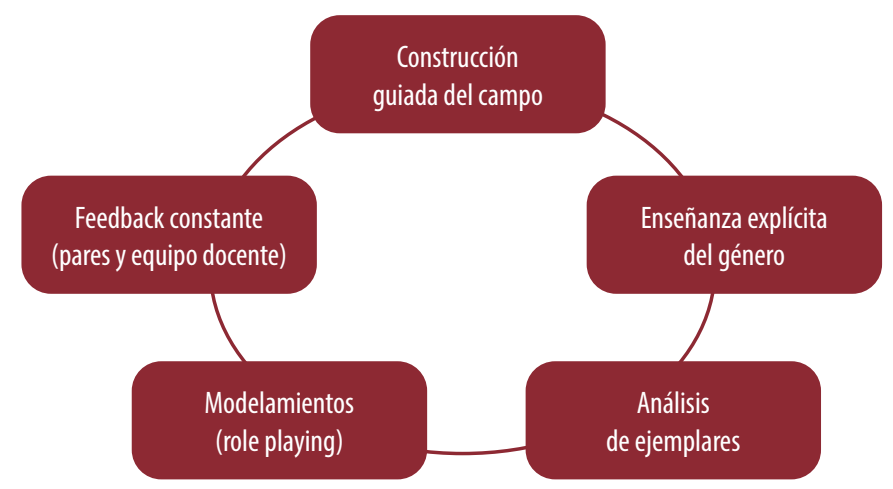

Figura 1. Diseño de secuencia pedagógica y recursos didácticos 
de la secuencia involucra el análisis de ejemplos de solemnes previas, con el fin de que los estudiantes integren con mayor profundidad las etapas del género. Un cuarto elemento considera modelamientos, basados en actividades prácticas en el contexto de aula como role playing (simulación de la instancia de solemne). Finalmente, un quinto elemento es el feedback constante por parte de pares, ayudante y equipo docente. La conjunción y recursividad de estos elementos permiten la enseñanza explícita del género, así como la acreditación constante de los aprendizajes por parte de los estudiantes. En la tabla 3 se puede ver la secuencia que despliega en el cronograma del programa de curso.

\section{Evaluación de la implementación}

Una vez realizada esta intervención se procedió a constatar su impacto a través de las opiniones de los estudiantes que cursaron el ramo. Para esto, se realizó una encuesta online sobre los principales aspectos trabajados en torno al género solemne y su posible transferencia a otros cursos de la carrera.

La primera pregunta, de naturaleza cerrada, buscó indagar en la percepción de pertinencia y de transferencia del dispositivo por parte de los estudiantes. Para esto, se les invitó a marcar su nivel de acuerdo (escala de Likert) con una serie de afirmaciones relativas al trabajo con el género solemne oral en el contexto del curso. Los resultados se indican en la tabla 4.

Entre los resultados destaca el alto nivel de acuerdo de los estudiantes con respecto a que en el curso se entregaron herramientas suficientes para estructurar respuestas en el contexto de una solemne oral ( $85,4 \%$ de los alumnos distribuidos en los niveles «muy de acuerdo» y «de acuerdo») y para desempeñarse paraverbalmente en dicho género ( $85,96 \%$ de los alumnos distribuidos en los niveles «muy de acuerdo» $\mathrm{y}$ «de acuerdo»). Lo anterior da cuenta de la existencia de una evaluación positiva bastante generalizada del trabajo que se realiza en base al género, a lo que se suma el nivel de acuerdo de los estudiantes con la utilidad de lo trabajado en el curso para enfrentarse a las solemnes orales de otros ramos de la carrera $(58,99 \%$ de los alumnos distribuidos en los niveles «muy de acuerdo» $\mathrm{y}$ «de acuerdo»). Pese a esto, este último resultado no parece ser tan contundente como el de los estímulos anteriores debido a que un $25,84 \%$ de los alumnos solo está parcialmente de acuerdo con lo anterior. Este último aspecto puede explicarse, en parte, por la baja percepción de semejanza en cuanto a la exigencia entre la solemne oral de este curso y la de las otras asignaturas ( $38,41 \%$ de los alumnos distribuidos en los niveles «muy de acuerdo» $\mathrm{y}$ «de acuerdo»).

Para profundizar en los datos presentados hasta aquí, a continuación se presentan los resultados obtenidos a partir del análisis de contenido cualitativo de las dos preguntas abiertas de la encuesta contestada por los estudiantes. 
Tabla 3. Cronograma del curso «Desarrollo de habilidades comunicativas para abogados»

\begin{tabular}{|c|c|}
\hline Fecha & Clase a clase \\
\hline \multicolumn{2}{|l|}{ MARZO } \\
\hline J-02 & Presentación del curso \\
\hline M-07 & Construcción preliminar del campo para el ensayo académico 1 y para la solemne oral 1 \\
\hline J-09 & Estrategias de lectura académica \\
\hline M-14 & Estrategias de lectura académica \\
\hline J-16 & Género solemne oral (variantes solemnes monológica y dialógica) \\
\hline M-21 & Género solemne oral (variantes solemnes monológica y dialógica) \\
\hline $\mathrm{J}-23$ & Elementos paraverbales y kinésicos de la comunicación oral académica \\
\hline M-28 & Actividad de ejercitación del género solemne oral (role playing) \\
\hline $\mathrm{J}-30$ & Actividad de ejercitación del género solemne oral (role playing) \\
\hline \multicolumn{2}{|l|}{ Abril } \\
\hline M-04 & Modelo de producción textual \\
\hline J-06 & Delimitación de un tema en el marco de un ensayo académico y planificación de una línea argumental \\
\hline M-11 & Evaluación oral 1 (género solemne oral) / trabajo autónomo y virtual \\
\hline J-13 & Evaluación oral 1 (género solemne oral) / trabajo autónomo y virtual \\
\hline M-18 & Evaluación oral 1: género solemne oral / trabajo autónomo y virtual \\
\hline $\mathrm{J}-20$ & Género ensayo académico (deconstrucción) / pasos del género y organización de la información \\
\hline M-25 & Género ensayo académico (deconstrucción) / pasos del género y organización de la información \\
\hline J-27 & Género ensayo académico (construcción conjunta) \\
\hline \multicolumn{2}{|l|}{ Mayo } \\
\hline M-02 & Género ensayo académico (construcción conjunta) \\
\hline $\mathrm{J}-04$ & Género ensayo académico (construcción individual) \\
\hline M-09 & Género ensayo académico (construcción individual) \\
\hline J-11 & Actividad de participación en clases: fin de construcción individual del género ensayo académico y revisión entre pares \\
\hline M-16 & Construcción preliminar del campo para el ensayo académico 2 y la solemne oral 2 \\
\hline J-18 & Género solemne oral - paso propiedades del fenómeno \\
\hline $\mathrm{M}-23$ & Práctica del género solemne oral \\
\hline J-25 & Práctica del género solemne oral - instrucciones trabajo autónomo virtual ensayo académico 2 \\
\hline $\mathrm{M}-30$ & Evaluación oral 2 (género solemne oral 2) / trabajo autónomo virtual \\
\hline \multicolumn{2}{|l|}{ JUNIO } \\
\hline $\mathrm{J}-01$ & Evaluación oral 2 (género solemne oral 2) / trabajo autónomo virtual \\
\hline M-06 & Evaluación oral 2 (género solemne oral 2) / trabajo autónomo virtual \\
\hline $\mathrm{J}-08$ & Actividad de participación en clases: revisión entre pares avance ensayo académico 2 y cierre del curso \\
\hline $\mathrm{J}-02$ & Entrega ensayo académico 2 \\
\hline
\end{tabular}


Tabla 4. Respuestas a la pregunta 1

\begin{tabular}{|c|c|c|c|c|c|c|c|c|c|c|}
\hline \multirow{2}{*}{ Descripción } & \multicolumn{2}{|c|}{$\begin{array}{l}\text { Muy de } \\
\text { acuerdo }\end{array}$} & \multicolumn{2}{|c|}{$\begin{array}{c}\text { De } \\
\text { acuerdo }\end{array}$} & \multicolumn{2}{|c|}{$\begin{array}{l}\text { Parcialmente } \\
\text { de acuerdo }\end{array}$} & \multicolumn{2}{|c|}{$\begin{array}{c}\text { En } \\
\text { desacuerdo }\end{array}$} & \multicolumn{2}{|c|}{$\begin{array}{c}\text { Muy en } \\
\text { desacuerdo }\end{array}$} \\
\hline & $\%$ & $\mathrm{~N}$ & $\%$ & N & $\%$ & $\mathrm{~N}$ & $\%$ & N & $\%$ & N \\
\hline $\begin{array}{l}\text { En el contexto del curso «Desarrollo de } \\
\text { habilidades comunicativas para abogados» se } \\
\text { me entregaron claros lineamientos acerca de } \\
\text { cómo estructurar una respuesta suficiente en } \\
\text { una solemne oral. }\end{array}$ & 51,69 & 92 & 33,71 & 60 & 9,55 & 17 & 2,81 & 5 & 2,25 & 4 \\
\hline $\begin{array}{l}\text { En el contexto del curso «Desarrollo de } \\
\text { habilidades comunicativas para abogados» } \\
\text { se me entregaron claros lineamientos acerca } \\
\text { de cómo desempeñarme paraverbalmente de } \\
\text { forma suficiente en una solemne oral. }\end{array}$ & 55,62 & 99 & 30,34 & 54 & 11,24 & 20 & 1,12 & 2 & 1,69 & 3 \\
\hline $\begin{array}{l}\text { Lo trabajado en torno al género solemne } \\
\text { oral en el contexto del curso me sirvió para } \\
\text { enfrentarme a las solemnes orales que he } \\
\text { tenido que enfrentar hasta el momento en } \\
\text { los otros cursos de este semestre. }\end{array}$ & 27,53 & 49 & 31,46 & 56 & 25,84 & 46 & 7,87 & 14 & 7,30 & 13 \\
\hline $\begin{array}{l}\text { La exigencia de las instancias de solemne de } \\
\text { los otros cursos fue similar a la exigencia de } \\
\text { la solemne oral de este curso. }\end{array}$ & 11,86 & 21 & 26,55 & 47 & 28,25 & 50 & 20,90 & 37 & 12,43 & 22 \\
\hline
\end{tabular}

\section{Percepción de manejo del género solemne oral}

Uno de los elementos más apreciados por los estudiantes en cuanto al dispositivo de enseñanza-aprendizaje del curso en torno al género solemne oral es que este, desde su percepción, los ayudó a manejar dicho género, es decir, a responder a las expectativas de desempeño en cuanto a esta práctica discursiva en el contexto de su trayectoria formativa. De esta manera, los estudiantes indican que lo trabajado en el curso los ha ayudado a enfrentarse a la instancia de la solemne oral: «Me ayudó a sentirme más preparada para las siguientes solemnes, a saber cómo se desarrollan y su estructura» (estudiante).

Según la percepción de los estudiantes, dicha preparación pasaría por diferentes aspectos. En primer lugar, el dispositivo sirvió como apoyo en su aproximación al género solemne oral. Así, indican que: «El trabajo realizado en clases abarcó la estructura y las características pertinentes a una solemne oral, por lo que se facilitó posteriormente el estudio y la rendición de la solemne» (estudiante). Uno de los aspectos relativos al manejo del género tiene relación con la ayuda que recibieron para estructurar las respuestas a las interrogantes que se plantean en el contexto de la solemne oral. Sobre este elemento, los alumnos indican que el curso «entrega una clara y correcta estructura organizativa para las respuestas» (estudiante), lo que es altamente apreciado entre ellos: «¡Muy buena iniciativa este curso! ¡Me sirvió para aprender a desarrollar una estructura y enfrentar mi prueba!» (estudiante). 
Cabe indicar que dicha apreciación podría estar relacionada con el carácter novedoso de la instancia solemne oral para ellos. Esto puede apreciarse en la evaluación positiva del dispositivo en relación con lo que tendrían que realizar en el contexto de su carrera: «Fue una buena práctica para conocer a qué nos enfrentaríamos» (estudiante); «Me sirvió como una actividad previa a las solemnes que tuve que rendir posteriormente en otros ramos. Me pude hacer una idea de cómo iban a ser» (estudiante).

Por otra parte, los alumnos indicaron que lo trabajado en el curso favoreció su expresión verbal y paraverbal en el contexto de realización de una solemne oral: «La manera de expresarnos verbalmente y paraverbalmente para enfrentar una solemne sirvió demasiado, ya que uno llega con muletillas o con gestos que son inadecuados para presentarse a una evaluación oral» (estudiante); «Ayudó para desenvolverse de mejor manera en las solemnes orales, desde el punto de vista oral, para así expresarse con mayor rigurosidad» (estudiante).

Otro elemento destacado por los estudiantes en cuanto a los apoyos recibidos en el curso para manejar este género fue la entrega de tips, datos y consejos para abordarlo: «Se entregaron tips útiles para desenvolverse en solemnes de otros ramos»; «Asimismo, la profesora nos dio tips para responder ante una evaluación oral, lo que me ayudó». Es importante indicar que los estudiantes tienden a no aclarar en qué consistieron dichos tips, aunque podría deducirse, a partir del conocimiento del dispositivo, que se refieren a elementos que explicitaron tanto el género como los elementos contextuales de su realización.

Como último aspecto relevado en las respuestas de los estudiantes en cuanto a la percepción de manejo del género, es posible observar su percepción de aumento de seguridad para enfrentar el género solemne oral. Es así como muchos de los alumnos subrayan que el curso incidió en su aumento de confianza, seguridad y tranquilidad, así como también en perder el miedo a esta instancia evaluativa. Lo anterior estaría vinculado con la percepción de preparación para esta: «Me dio confianza, me preparó y me dejó conforme» (estudiante). Pero también es en el contexto de este curso en el que los alumnos se enfrentan por primera vez a una solemne oral: «Al ser una instancia previa a las solemnes, me ayudó a prepararme y no sentirme tan nerviosa para el resto y para saber cómo funcionaba la dinámica» (estudiante).

A modo de cierre, cabe indagar en ciertos aspectos presentes en el discurso de los estudiantes relacionados con la conciencia del género. De alguna manera, este curso, que trabaja las habilidades escritas y orales desde un enfoque de género, hizo que dicho constructo se hiciera presente en las percepciones de los estudiantes. Es así como en sus respuestas es posible observar reflexiones que dan cuenta del estrecho vínculo entre forma y contenido: «Por muy bien que uno sepa los contenidos, es fundamental saber cómo expresarlos y eso precisamente nos enseñó el curso» (estudiante). Además, se observa la noción de que las características del desempeño oral y escrito 
son situadas: «Se facilitó la comprensión y aprendizaje del ordenamiento dentro del discurso oral o de una prueba oral como son las solemnes o exámenes» (estudiante).

\section{Percepción de preparación para enfrentar el género solemne oral}

Otro de los aspectos que destacan los estudiantes en su evaluación del trabajo del género solemne en el contexto del dispositivo es la percepción de preparación para el manejo del género: «Aspectos favorables sobre el curso son la preparación como novata que tuve para desenvolverme en una solemne» (estudiante).

Dicha percepción de preparación suele estar acompañada de otros aspectos. Uno de estos corresponde a la instrucción explícita de la estructura del género solemne oral: «Las habilidades aprendidas resultaron ser muy útiles al momento de saber cómo enfrentarme a una solemne de otra asignatura, por lo que me sirvieron para estar más tranquila y para dar respuestas claras y estructuradas» (estudiante). Un segundo elemento consiste en la entrega de información relativa a aspectos contextuales de la práctica evaluativa en la que se inserta este género, como la conformación de una comisión evaluadora integrada por profesores y ayudantes, la forma de entrega de los estímulos, entre otros aspectos. La explicitación de dichos aspectos le permitió a los alumnos conocer multidimensionalmente la realización del género, lo que también resulta favorecedor de su preparación para enfrentar dicha práctica discursiva: «En general, el haber desarrollado una solemne en el curso me ayudó a no enfrentarme a este tipo de prueba con tantas dudas sobre qué podía pasar»; «al ser una instancia previa a las solemnes me ayudó para prepararme y no sentirme tan nerviosa para el resto y para saber cómo funcionaba la dinámica» (estudiante). Por último, los estudiantes destacan el trabajo de los aspectos de expresión verbal y paraverbal como parte de los aprendizajes que los hacen sentirse preparados para enfrentar el género: «Lo trabajado en clases fue favorable, ya que se enseñaron diversas formas de enfrentarse a una solemne, desde la postura, el uso del lenguaje y cómo estructurar correctamente una respuesta sólida» (estudiante).

\section{Percepción de transferencia del manejo del género solemne oral}

Aparejada a las percepciones desarrolladas, de forma recurrente aparece en las respuestas de los estudiantes la percepción de que aquello que aprendieron en el curso sobre el manejo del género solemne oral ha afectado positivamente su desempeño en las solemnes orales de otras instancias de su carrera:

Me gustó cómo el curso me ayudó a enfrentar de mejor manera mis solemnes en los otros ramos. Fue un buen acercamiento a lo que me enfrentaría en la realidad. Es un ramo muy amigable para comenzar a introducirse en el mundo del derecho (estudiante). 
Esta percepción de transferencia se hizo presente constantemente en el discurso de los estudiantes a partir de la indicación explícita de que lo aprendido en relación con este género ha impactado en su desempeño en otros cursos:

Favoreció mi expresión oral para el resto de las solemnes y me ayudó a organizar una estructura mental para mis respuestas (estudiante).

Las herramientas entregadas son útiles para la actitud adecuada que se debe tener al momento de enfrentarse a una solemne (estudiante).

Nos entrega estructura y consejos para los demás ramos (estudiante).

Me permitió conocer los tips necesarios para estructurar una buena respuesta en las solemnes de las asignaturas propias de la especialidad (estudiante).

Ayudó para desenvolverse de mejor manera en las solemnes orales, desde el punto de vista oral, para así expresarse con mayor rigurosidad (estudiante).

Este curso permite la práctica para las solemnes en los otros ramos, lo que es muy útil, ya que siendo novata uno no sabe cómo serán y este ramo ayuda mucho para dar una buena prueba (estudiante).

Se abarca el tema de la prueba solemne de forma simple, lo cual facilita su comprensión,y ayuda a recordar más fácilmente lo aprendido cuando uno se enfrenta a solemnes en otros ramos (estudiante).

Las habilidades aprendidas resultaron ser muy útiles al momento de saber cómo enfrentarme a una solemne de otra asignatura, por lo que sirvieron para estar más tranquila, para dar respuestas claras y estructuradas (estudiante).

En relación con este resultado, cabe indicar que la percepción de transferencia de los estudiantes resulta altamente positiva en términos de la evaluación del dispositivo. Esto se debe a que los alumnos consideran que lo aprendido en el curso es transferible a otras asignaturas, lo que coincide con uno de los aspectos del objetivo de aprendizaje general del dispositivo: «Conocer e incorporar estrategias de lectura, escritura y oralidad necesarias para enfrentar los desafíos propios del estudio del derecho en los primeros años». Pese a lo anterior, cabe considerar que, para tener una idea más acabada del nivel de transferencia alcanzado por los estudiantes a partir de este dispositivo, se requiere desarrollar otras investigaciones que indaguen en el desempeño real de los estudiantes intervenidos, así como en la percepción de los docentes disciplinares, entre otros aspectos.

\section{Necesidad de mayor relación/semejanza entre la solemne oral trabajada en el curso y las de las otras asignaturas de la carrera}

Pese a la alta valoración del trabajo relativo a este género en el contexto del curso, y como es posible apreciar en los resultados de la pregunta 1 de la encuesta, hay estudiantes que indican la necesidad de que lo presentado como género solemne oral 
resulte más semejante a lo que se trabaja y solicita como solemne oral en otras asignaturas de derecho.

Para esto, los alumnos sugieren cambios en relación con la estructura del género: «Que haya más de relación entre esta estructura de solemne con las demás solemnes» (estudiante). Asimismo, se menciona lo siguiente: «Pienso que se debería adecuar mejor la forma de hacer las preguntas, ya que normalmente en las solemnes de otros cursos las respuestas deben ser directas y no se da tiempo para prepararlas» (estudiante).

También, proponen aumentar el nivel de exigencia con el que se evalúa la solemne oral de forma de alcanzar un mayor grado de similitud de los que se trabaja en el curso con lo que se les exige en las otras asignaturas: «Tal vez, la exigencia en la solemne es lo más mejorable, ya que en los cursos de derecho es muy superior» (estudiante). Además, mencionan que debe alinearse más con las expectativas de desempeño de los profesores disciplinares: «El único problema es que las solemnes de los otros ramos no son parecidas a la solemne de let (nombre que los estudiantes utilizan para identificar el curso). Al profesor le interesa que diga la materia más que si mi vocabulario es $100 \%$ formal, por ejemplo» (estudiante).

Dichas sugerencias, de alto valor para el equipo docente, destacan varios aspectos relevantes. El primero de ellos es identificar las limitantes disciplinares con las que cuentan los expertos en lengua que trabajan en pos de la alfabetización académico y disciplinar, así como las de ciertos dispositivos de alfabetización. Al ser el diseñador y mediador de este dispositivo un experto en lengua y no un disciplinar del área del derecho, los objetivos del proceso de enseñanza-aprendizaje y los focos de este varían de forma significativa. Es así como mientras el docente disciplinar trabaja en pos de la apropiación de conocimiento disciplinar y de inserción al campo por parte de los alumnos, el experto en lengua tiene como foco que los estudiantes desarrollen habilidades que les permitan insertarse en una comunidad discursiva particular. Dicha varianza afectaría la forma de trabajo del género, lo que no es bien recibido por parte de los alumnos.

Por otra parte, los aspectos a mejorar desde la mirada de los alumnos permiten evidenciar la alta variabilidad del género solemne oral en el contexto de la carrera de Derecho, al menos en la institución que constituyó el campo de estudio. Si bien se llegó a esta conclusión a partir de la indagación realizada que permitió detectar solemnes dialógicas y monológicas, entre otras diferencias esta investigación tuvo como limitante el que solo se tomaran muestras de las solemnes orales realizadas en dos asignaturas.

Por último, es importante indicar que, pese a que el equipo investigador y docente tiene conciencia de la necesidad de trabajar las varianzas del género en el curso, solo es posible hacerlo en parte debido a las constricciones temporales y a que el curso cuenta con otros objetivos de aprendizaje que coexisten con el de desarrollar habilidades para enfrentar una solemne oral. 


\section{Recursos didácticos para la enseñanza-aprendizaje del género solemne oral}

Un último aspecto relevado en la evaluación de los estudiantes del dispositivo tiene relación con los recursos didácticos desplegados en el curso en función de la enseñanza-aprendizaje del género solemne oral. En dicho contexto, los alumnos destacaron las prácticas relativas a la evaluación, al modelamiento a partir de ejemplos reales, a la existencia de instancias de ejercitación, así como también a que este curso tuviera la primera instancia de solemne en el contexto de la carrera.

En cuanto al proceso evaluativo, los estudiantes valoraron muy positivamente la disponibilidad y entrega de instrumentos de evaluación claros que explicitaran las expectativas de desempeño: «Se entrega una estructura clara y una rúbrica transparente, así sabemos qué debemos cumplir para desarrollar una buena solemne» (estudiante). Por otra parte, se señala que: «Además, me pareció muy bueno que la rúbrica de evaluación siempre fuese de conocimiento público, ya que así teníamos lineamientos claros para desempeñar una buena solemme oral» (estudiante).

Como segundo elemento relativo a la pedagogía del género, los alumnos valoraron muy positivamente el trabajo a partir de ejemplos reales de solemnes orales realizadas por estudiantes en semestres anteriores de la carrera: «Fue muy positivo que hayamos revisado solemnes de años anteriores» (estudiante); «fue una buena explicación de la forma en la que se estructura una solemne, vimos en clases hasta solemnes pasadas que nos sirvieron de ejemplo para poder estructurar nuestras propias pruebas» (estudiante).

Un tercer aspecto relevado por los estudiantes como propiciador de su proceso de enseñanza-aprendizaje del género fue contar con instancias de ejercicio que consistían en sesiones de modelamiento voluntario de este como una instancia formativa no calificada: «Las técnicas y consejos fueron muy útiles, especialmente los ensayos de solemne» (estudiante). Además, se menciona que:

La ejemplificación de las modalidades que efectivamente corresponden a la ocasión de una solemne resultaba de suma utilidad, en especial cuando se solicitaba la participación y la simulación de una solemne junto con el uso de una rúbrica, ya que permitía identificar de manera clara cada parámetro a mejorar (estudiante).

Es importante indicar que, junto con esta evaluación positiva de las instancias de ejercitación, los estudiantes indicaron la necesidad de aumentarlas para alcanzar un mayor dominio del género y tanto para ganar mayor seguridad como tranquilidad para enfrentarlo. Es así como, en relación con los aspectos mejorables del curso, los alumnos propusieron hacer «más «simulacros» de solemne para estar más preparados y saber cómo controlarse al momento de responder» (estudiante); «quizá aumentar las actividades orales, no exclusivamente con el objetivo de una evaluación, sino 
que en pro de asimilar la postura que debemos adoptar y a su vez reducir el nerviosismo de una exposición» (estudiante).

Por último, resultó especialmente valorado por los estudiantes el que la primera instancia de enfrentamiento a una solemne oral se diera en el contexto de este curso. Cabe indicar que el dispositivo consideró, en el diseño de la secuencia pedagógica, que se trabajara y evaluará este género antes de que se diera inicio a la primera ronda de solemnes de los cursos disciplinares de derecho, de manera que los estudiantes contaran con herramientas para enfrentarse a dichas evaluaciones.

Con respecto al punto anterior, los alumnos relevan positivamente que la primera solemne oral haya sido en el contexto de este dispositivo debido a que, fuera del contexto de los cursos disciplinares, no había tanta presión: «Fue muy bueno tener la posibilidad de responder una solemne sin mucha presión previo a tener cualquier otra. Fue sumamente agradable, ya que me permitió relacionarme mejor con el formato» (estudiante).

Por otra parte, el contar con una instancia previa a las solemnes orales de los otros cursos en la que pudieran conocer y ejercitar el género fue valorado por los estudiantes, ya que les permitió hacerse una idea y bajar sus niveles de ansiedad ante la evaluación:

Creo que, personalmente, me ayudó a perderle el miedo a una prueba oral, pude soltarme un poco antes de enfrentar las de los otros ramos (estudiante).

Aprendimos a usar palabras académicas y a hablar en público. Sirvió mucho que fuera la primera solemne antes de las de los ramos de derecho (estudiante).

Me sirvió como una actividad previa a las solemnes que tuve que rendir posteriormente en otros ramos. Me pude hacer una idea de cómo iban a ser (estudiante).

Me sirvió, ya que fue la primera instancia oral en la universidad. En ese sentido, fue bueno haber partido con esta solemne antes que con otras (estudiante).

A partir de la revisión y del análisis de las categorías hasta aquí desarrolladas que fueron producto del análisis de contenido cualitativo de las respuestas de los estudiantes a la encuesta, es posible dar cuenta de la percepción de manejo del género solemne oral por parte de los estudiantes, el que sería producto, en parte al menos, de lo trabajado en el contexto del curso. Dicho manejo afectaría positivamente la percepción de seguridad a la hora de enfrentarse a la realización del género en el curso, así como en el contexto de otras asignaturas de la carrera. Pese a lo anterior, desde la mirada de los estudiantes, es necesario seguir trabajando para lograr una mayor semejanza tanto entre lo que se trabaja y exige en el curso como con lo que se presenta como solemne oral en los otros cursos de la malla curricular de la carrera. 


\section{Conclusión}

La intervención descrita busca enfatizar la necesidad de que toda iniciativa o propuesta en el desarrollo de habilidades comunicativas académicas disciplinares se plantee desde una base teórica y metodológica explícita, que considere tanto el proceso de diseño, la implementación y el impacto de la propuesta realizada. Desde esta mirada, esta investigación da cuenta de la descripción del género solemne oral, del diseño de dispositivos específicos para su enseñanza en el aula y de la posterior evaluación por parte de los estudiantes sobre el aprendizaje de este género.

Otro aspecto fundamental de las intervenciones en alfabetización académica es el rol que juegan todos los actores involucrados en la comunidad discursiva específica de la disciplina: directivos, docentes y estudiantes. Se necesita del trabajo de toda la comunidad docente y de las autoridades para que los estudiantes puedan adentrarse en las características discursivas de su campo y convertirse en miembros de su disciplina. Se requiere de la instalación de una conciencia de cómo y cuánto permea el aprendizaje en esta área, como en todas, el manejo de las formas discursivas propias de ella y, por otra parte, del desarrollo de estrategias didácticas orientadas al desarrollo de habilidades comunicativas situadas que propician el desarrollo de conocimiento especializado.

En cuanto a la evaluación de la enseñanza específica del género, la percepción de transferencia de los estudiantes resulta muy positiva tanto para ellos como en términos de evaluación del dispositivo diseñado en el marco del presente proyecto de investigación-acción. En cuanto a lo primero, y según la literatura especializada del campo de la enseñanza-aprendizaje, un aumento en el autoconcepto del estudiante como escritor en su propia disciplina, que en este caso estaría vinculado con la percepción de manejo del género y con su transferencia, es un propiciador del aprendizaje.

A modo de proyección, es necesario consolidar los resultados de este estudio para medir el nivel de transferencia alcanzado por los estudiantes a partir de este dispositivo. Para esto, es necesario desarrollar otras investigaciones que indaguen en el desempeño por parte de los estudiantes en otros contextos de producción de géneros orales académicos, así como en la percepción de los docentes disciplinares en torno a las habilidades comunicativas que se deben desarrollar en el marco de la formación en derecho, entre otros aspectos.

\section{Referencias}

Andino, Alexandra, Hayk Paronyan, Alberto Santillán y Cristoval Rey (2019). «La Veeduría: Herramienta pedagógica dirigida a estudiantes de la carrera de Derecho que presentan limitaciones en el desarrollo de la oralidad». Dilemas Contemporáneos: Educación, Política y Valores, 6: 1-20. Disponible en bit.ly/31B52BP. 
Álvarez, Graciela (2008). «La enseñanza del discurso jurídico oral y escrito en la carrera de Abogacía». Revista sobre enseñanza del Derecho, (6) 11: 137-148. Disponible en bit.ly $/ 35 \mathrm{FBPjH}$.

BARBeIRo, Luís. (2003). Escrita: Construir a aprendizagem. Braga: Universidade do Minho.

BARDin, Laurence. (1991). Análisis de contenido. Madrid: Akal.

Peter Reason y Bradbury, Hilary. (2008). Handbook of action research. Londres: SAGE.

Coloma, Rodrigo y Claudio Agüero. (2012). «Los abogados y las palabras: una propuesta para fortalecer competencias iniciales en los estudiantes de derecho». Revista de derecho (Coquimbo), 19 (1): 39-69. Disponible en bit.ly/3f6cSAS.

Condon, William y Diane Kelly-Riley. (2004). «Assessing and teaching what we value: The relationship between college-level writing and critical thinking abilities». Assessing Writing, 9 (1): 56-75. Disponible en bit.ly/3nrl $4 \mathrm{P}_{2}$.

Cuenca, Maritza y Alexandra Andino (2020). «La evaluación del desarrollo de la competencia comunicativa oral de los profesionales de derecho». Revista Mikarimin, (6): 1-10. Disponible en bit.ly/2H7koir.

Doumont, Jean Luc (2009). Trees maps and theorems. Effective communication for rational minds. Kraainem: Principae.

Dreyfus, Shoshana, Sally Humphrey, Ahmar Mahbood y James Martin (2016). Genre Pedagogy in Higher Education. The SLATE Project. Londres: Palgrave MacMillan.

IвÁÑEz, Romualdo y Cristian González (2017). Alfabetización disciplinar en la Formación Inicial Docente. Leer y escribir para aprender. Valparaíso: Universitarias de Valparaíso.

Israel, Barbara, Amy Schulz, Edith Parker y Adam Becker (2008). «Critical Issues in Developing and Following Community-Based Participatory Research Principles». En Meredith Minkler y Nina Wallerstein (editores), Community-Based Participatory Research for Health (pp. 47-62). San Francisco: Jossey-Bass.

JARPA, Marcela y Juan Satt (2017). «Géneros académicos en la Formación Inicial Docente: la variación disciplinar y las tareas de lectura y escritura». En Romualdo Ibáñez, Romualdo y Cristian González (editores), Alfabetización disciplinar en la Formación Inicial Docente. Leer y escribir para aprender (pp. 43-64). Valparaíso: Universitarias de Valparaíso.

JEwitT, Carey (2006). Technology, literacy and learning: A multimodal approach. Londres: Routledge.

KLEIN, Perry y Pietro Boscolo (2016). «Trends in research on writing as a learning activity». Journal of Writing Research, 7 (3):311-350. DOI: 10.17239/jowr-2016.07.03.01

LEA, Mary y Brian Street (2006). «The «academic literacies» model: Theory and applications». Theory into practice, 45 (4): 368-377. Disponible en bit.ly/3f5rRev. 
Martin, Jim. (1992). «Genre and literacy-modeling context in educational linguistics». Annual review of applied linguistics, (13): 141-172. DOI: 10.1017/ So267190500002440

Martin, Jim y David Rose. (2008). Genre relations: Mapping culture. Londres: Equinox.

Miras, Mariana (2000). «La escritura reflexiva. Aprender a escribir y aprender acerca de lo que se escribe». Infancia y aprendizaje, 23 (89): 65-8o. DOI: 10.1174/021037000760088099.

PAlomo, Diego (2004). «Y ahora, tras la experiencia procesal penal ¿la oralidad al proceso civil?: Algunas claves y criterios a seguir». Ius et Praxis, (10) 2: 225-265. Disponible en bit.ly/3pALX 56 .

PoyATOS, Fernando (2003). «La comunicación no verbal: algunas de sus perspectivas de estudio e investigación». Revista de Investigación Lingüística, (6) 2: 67-83. Disponible en bit.ly/3lI4XfK.

Rose, David y Jim Martin (2012). Learning to write, reading to learn: Genre, knowledge and pedagogy in the Sydney School. Sheffield: Equinox.

Schmandt-Besserat, Demise y Michael Erard (2008). «Origins and forms of writing». En Charles Bazerman, Handbook of research on writing: History, society, school, individual, text (pp.7-22). Santa Bárbara: Lawrence Elbaum.

SwALES, John (1990). Genre analysis: English in academic and research settings. Cambridge: Cambridge University Press.

URRejola, Karen y Vidal, Margarita (en prensa). «Opciones teóricas y didácticas en el programa PLEA para la enseñanza de la alfabetización disciplinar inicial». En Centros y Programas en Latinoamérica: Opciones teóricas y pedagógicas para la enseñanza de la escritura disciplinar. Colorado: WAC Clearinghouse.

\section{Sobre los autores}

María Verónica Sánchez Gibbons es licenciada en Letras Hispánicas y magíster en Psicología Educacional por la Pontificia Universidad Católica de Chile. Su correo electrónico es mvsanche@uc.cl. (1) https://orcid.org/oooo-0002-3020-566X.

Karen Urrejola Corales es licenciada en Letras Hispánicas y magíster en Lingüística por la Pontificia Universidad Católica de Chile. Su correo electrónico es kuc@uc.cl. (D) https://orcid.org/oooo-0001-7518-3428.

Nelson BeCERRa Rojas es profesor de Lenguaje y Literatura, y magíster en Lingüística Aplicada por la Pontificia Universidad Católica de Chile, Su correo electrónico es nelson.becerra@uc.cl. (D) https://orcid.org/oooo-0003-3000-3141. 
